\title{
Business Processes Contextualisation via Context Analysis
}

\author{
Jose Luis de la Vara ${ }^{1}$, Raian $\mathrm{Ali}^{2}$, Fabiano Dalpiaz ${ }^{2}$, Juan Sánchez ${ }^{1}$, \\ and Paolo Giorgini ${ }^{2}$ \\ ${ }^{1}$ Centro de Investigación en Métodos de Producción de Software \\ Universidad Politécnica de Valencia, Spain \\ \{jdelavara, jsanchez\}@ pros.upv.es \\ ${ }^{2}$ Department of Information Engineering and Computer Science \\ University of Trento, Italy \\ \{raian.ali, fabiano.dalpiaz, paolo.giorgini\}@disi.unitn.it
}

\begin{abstract}
Context-awareness has emerged as a new perspective for business process modelling. Even though some works have studied it, many challenges have not been addressed yet. There is a clear need for approaches that (i) facilitate the identification of the context properties that influence a business process and (ii) provide guidance for correct modelling of contextualised business processes. This paper addresses this need by defining an approach for business process contextualisation via context analysis, a technique that supports reasoning about context and discovery of its relevant properties. The approach facilitates adequate specification of context variants and of business process execution for them. As a result, we obtain business processes that fit their context and are correct.
\end{abstract}

Keywords: business process modelling, context-awareness, business process contextualisation, context analysis, correctness of business process models.

\section{Introduction}

Traditional approaches for business process modelling have not paid much attention to the dynamism of the environment of a business process. However, business processes are executed in an environment in which changes are usual, and modelling perspectives that aim to represent and understand them are necessary.

Context-awareness has recently appeared as a new perspective for business process modelling to meet this need [3]. It is expected to improve business process modelling by explicitly addressing fitness between business processes and their context. The context of a business process is the set of environmental properties that affect business process execution. Therefore, these properties should be taken into account when designing a business process. If context is analysed when modelling a business process, then identification of all its variants (relevant states of the world in which the business process is executed) and definition of how the business process should be executed in them are facilitated. 
Some works have contributed to the advance of context-aware business process modelling by addressing issues such as context-aware workflows [4], general principles (e.g. [3]) and modelling of context effect (e.g. [2]). However, research on this topic is still at an initial stage and many challenges have not been addressed yet. This paper aims to advance in research on context-aware business process modelling by dealing with two of these challenges: 1) provision of techniques for determination of the relevant context properties that influence a business process, and; 2) provision of mechanisms and guidance for correct business process contextualisation.

The objectives of the paper are to determine how business process context can be analysed, how it can influence business processes, how to create contextualised business process models, and how to guarantee their correctness. These objectives are achieved by defining an approach for business process contextualisation via context analysis [1], which is a technique that aims to support reasoning about context and discovery of contextual information to observe. Context analysis is adapted in the paper for analysis of business process context.

The approach provides mechanisms and guidance that can help process designers to reason about business process context and to model business processes that fit their context and are correct. Context properties and variants are analysed in order to determine how they influence a business process, to guarantee that a business process is properly executed in all its context variants, and to correctly model contextualised business processes.

The next sections present the approach and our conclusions, respectively.

\section{Approach Description}

The approach consists of four stages (Fig. 1): modelling of initial business process, analysis of business process context, analysis of context variants and modelling of contextualised business process. First, an initial version of the business process that needs to fit its context is modelled. Next, the rest of stages have to be carried out while relevant context variations (changes) are found and they are not represented in the business process model. Relevant context variations influence the business process and imply that business process execution has to change.

If a context variation is found, then business process context is analysed to find the context properties that allow process participants to know if a context variant holds. A context analysis model is created, and context variants of the business process are then analysed. Finally, a contextualised business process model is created on the basis of the final context variants and their effect on the business process.

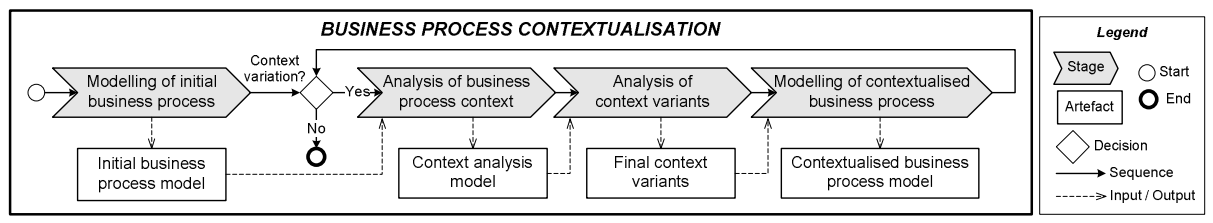

Fig. 1. Business process contextualisation 
As a running example, product promotion in a department store is used (Fig. 2). The business process has been modelled with BPMN, and it does not reflect context variations such as the fact that customers do not like being addressed if they are in a hurry. The paper focuses on contextualisation of the task "Find potential buyer".

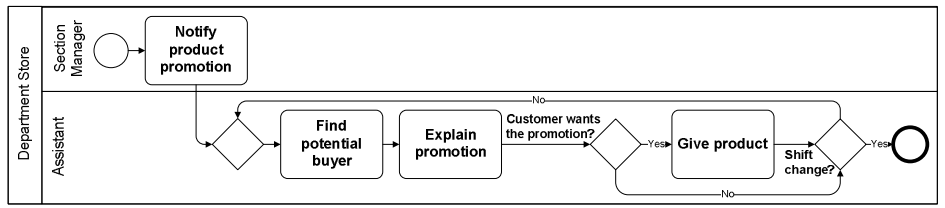

Fig. 2. Initial business process model

\subsection{Analysis of Business Process Context}

Business process context is analysed in the second stage of the approach. This stage aims to understand context, to reason about it and to discover the context properties that influence a business process. For these purposes, context analysis (which has been presented in the requirements engineering field) has been adapted for analysis of business process context. Further details about context analysis can be found in [1].

Context is specified as a formula of world predicates, which can be combined conjunctively and disjunctively. World predicates can be facts (they can be verified by a process participant) or statements (they cannot be). The truth value of a statement can be assumed if there is enough evidence to support it. Such evidence comes from another formula of world predicates that holds.

A context is judgeable if there exists a formula of facts that supports it, and thus implies it. Identifying a judgeable context can be considered the main purpose of this stage. The facts of the formula correspond to the context properties that characterise the context and its variants, and their truth values influence business process execution. A context analysis model (Fig. 3) is created to facilitate reasoning about business process context and discovery of the facts of the formula that implies it.

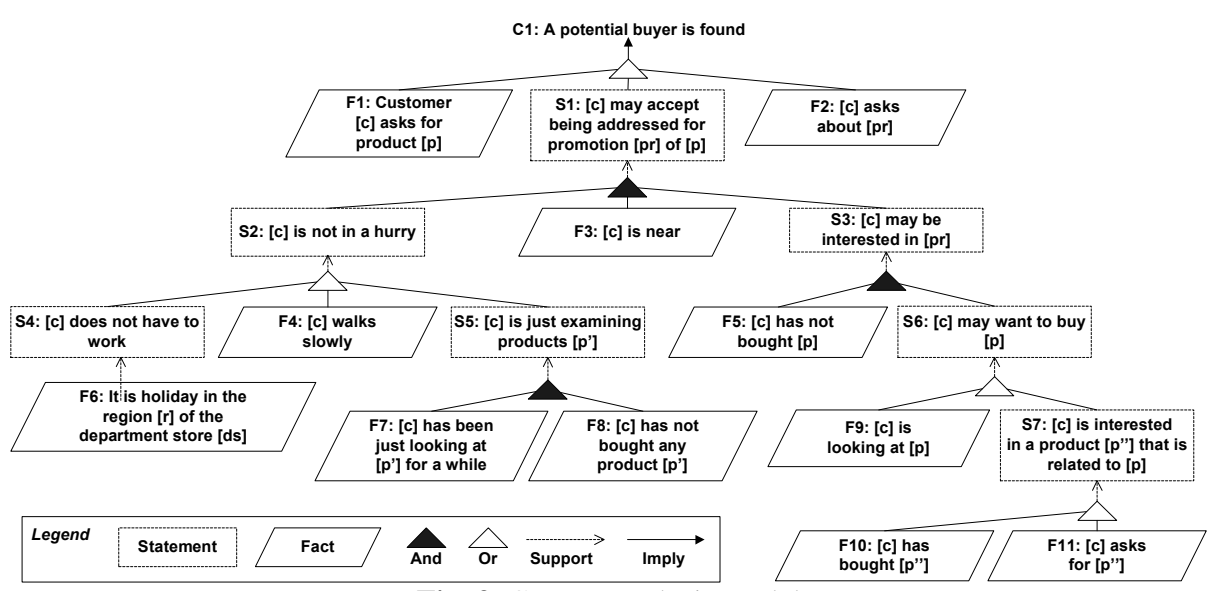

Fig. 3. Context analysis model 


\subsection{Analysis of Context Variants}

The main purposes of this stage are to adequately define the (final) context variants of a business process and that they allow correct business process contextualisation. A context variant corresponds to a set of facts whose conjunction implies a context. Fig. 4 shows the eleven initial context variants for $\mathrm{C} 1$, which is analysed in Fig. 1.

\begin{tabular}{|c|c|c|}
\hline Initial Context Variants & \multirow[t]{11}{*}{$\rightarrow$} & Final Context Variants \\
\hline$\{\mathrm{F} 1\}$ & & CV1: $\{F 1\}$ \\
\hline$\{\mathrm{F} 2\}$ & & CV2: $\{F 2\}$ \\
\hline$\{\mathrm{F} 3, \mathrm{~F} 4, \mathrm{~F} 5, \mathrm{~F} 9\}$ & & CV3: $\{$ F3 $\rightarrow($ F4, F5, F9) $\}$ \\
\hline$\{\mathrm{F} 3, \mathrm{~F} 4, \mathrm{~F} 5, \mathrm{~F} 10\}$ & & CV4: $\{\mathrm{F} 3 \rightarrow(\mathrm{F} 4, \mathrm{~F} 5, \mathrm{~F} 10)\}$ \\
\hline$\{\mathrm{F} 3, \mathrm{~F} 4, \mathrm{~F} 5, \mathrm{~F} 11\}$ & & CV7: $\{\mathrm{F} 4, \mathrm{~F} 11 \rightarrow \mathrm{F} 5\}$ \\
\hline$\{F 3, F 5, F 6, F 9\}$ & & CV5: $\{\mathrm{F} 6 \rightarrow \mathrm{F} 3 \rightarrow(\mathrm{F} 5, \mathrm{~F} 9)\}$ \\
\hline$\{\mathrm{F} 3, \mathrm{~F} 5, \mathrm{~F} 6, \mathrm{~F} 10\}$ & & CV6: $\{\mathrm{F} 6 \rightarrow \mathrm{F} 3 \rightarrow(\mathrm{F} 5, \mathrm{~F} 10)\}$ \\
\hline$\{F 3, F 5, F 6, F 11\}$ & & CV8: $\{\mathrm{F} 6 \rightarrow \mathrm{F} 11 \rightarrow \mathrm{F} 5\}$ \\
\hline$\{F 3, F 5, F 7, F 8, F 9\}$ & & CV9: $\{$ F3 $\rightarrow(F 7, F 8, F 9)\}$ \\
\hline$\{F 3, F 5, F 7, F 8, F 10\}$ & & CV10: $\{$ F7, F11 $\rightarrow$ F8 $\}$ \\
\hline$\{\mathrm{F} 3, \mathrm{~F} 5, \mathrm{~F} 7, \mathrm{~F} 8, \mathrm{~F} 11\}$ & & \\
\hline
\end{tabular}

Fig. 4. Context variants

Correctness of business processes is usually related to its soundness [5]. For business process executions that are defined from context variants, two situations can impede soundness of a contextualised business process. The first one is that a context variant contains conflicting facts. The second situation is to follow a sequence of fact verifications that will not allow a business process instance to be finished.

These situations are avoided by analysing the context variants. For this purpose, a table is created to specify the relationships between facts. The table also aims to obtain context variants whose sets of facts are the minimum ones. An example is shown in Table 1, which specifies the relationships between the facts of the initial context variants of Fig. 4. The relationships are specified as follows.

Given a pair of facts Fr (fact of a row) and Fc (fact of a column), their relationship can be: ' $\mathrm{X}$ ' (no context variant contains Fr and Fc together); 'Pr' (Fr verification will precede Fc verification); 'Pc' (opposite to 'Pr'); ' $\mathrm{Kr}$ ' (Fr truth value will be known before Fc verification); 'Kc' (opposite to ' $\mathrm{Kr}$ '); 'Ur' (Fr is always true when the Fc is true, thus Fr verification will be unnecessary when Fc is true); 'Uc' (opposite to 'Ur'); 'C' (Fr and Fc are conflicting); '-' (no relationship exists).

Finally, context variants are refined by specifying sequence of fact verification $(' \rightarrow$ ') and removing conflicting variants and unnecessary facts (Fig. 4).

Table 1. Relationships between facts

\begin{tabular}{|c|c|c|c|c|c|c|c|c|c|c|}
\hline & F11 & $\mathrm{F} 10$ & F9 & F8 & F7 & F6 & F5 & F4 & F3 & F2 \\
\hline $\mathrm{F} 1$ & $X$ & $X$ & $X$ & $X$ & $X$ & $X$ & $X$ & $X$ & $X$ & $X$ \\
\hline $\mathrm{F} 2$ & $\mathrm{X}$ & $X$ & $X$ & $\mathrm{X}$ & $X$ & $X$ & $X$ & $X$ & $X$ & \\
\hline F3 & $\overline{U r}$ & $\mathrm{Pr}$ & $\mathrm{Pr}$ & $\mathrm{Pr}$ & $\mathrm{Pr}$ & $\mathrm{Kc}$ & $\mathrm{Pr}$ & $\mathrm{Pr}$ & & \\
\hline F4 & - & - & - & $\bar{X}$ & $X$ & $X$ & - & & & \\
\hline F5 & $\mathrm{Pc}$ & - & - & Ur & - & Kc & & & & \\
\hline F6 & $\mathrm{Kr}$ & $\mathrm{Kr}$ & $\mathrm{Kr}$ & $\bar{X}$ & $X$ & & & & & \\
\hline F7 & - & - & - & - & & & & & & \\
\hline F8 & $\mathrm{Pc}$ & C & - & & & & & & & \\
\hline $\mathrm{F9}$ & $X$ & $X$ & & & & & & & & \\
\hline F10 & $\bar{X}$ & & & & & & & & & \\
\hline
\end{tabular}




\subsection{Modelling of Contextualised Business Process}

A contextualised business process is modelled on the basis of its final context variants. The first step is determination of the tasks that will be part of the business process. They can correspond to: 1) tasks of the initial business process model that are not influenced by context); 2) tasks that are defined from refinement of the tasks of the initial business process model (e.g. "Address customer" refines "Find potential buyer"), and; 3) tasks that make facts true (e.g. "Approach customer" makes F3 true). If a task of the latter type is executed when a given fact is false, then the fact turns into true. These facts are called manageable.

Table 2. Relationships between tasks and facts

\begin{tabular}{|l|c|c|c|c|c|c|c|c|c|c|c|}
\cline { 2 - 11 } \multicolumn{1}{c|}{} & F1 & F2 & F3 & F4 & F5 & F6 & F7 & F8 & F9 & F10 & F11 \\
\hline T1: Approach customer & U & U & M & - & - & - & - & - & - & - & U \\
\hline T2: Address customer & U & U & Sc & Sc1 & Sc1 & Sc1 & Sc1 & Sc1 & Sc1 & Sc1 & U \\
\hline
\end{tabular}

Once tasks are determined, a table is created to specify their relationships with the facts of the final context variants. An example is shown in Table 2. The relationships are specified as follows.

Given a fact $F$, a set of facts $\varphi$ and a task T, their relationship can be: 'M' ( $T$ allows $\mathrm{F}$ to be manageable); ' $\mathrm{U}$ ' ( $\mathrm{T}$ execution will be unnecessary if $\mathrm{F}$ is true); 'Sc' ( $\mathrm{T}$ execution will succeed $\mathrm{F}$ verification); ' $\mathrm{ScX}$ ' (where ' $\mathrm{X}$ ' is a number; $\mathrm{T}$ execution will succeed verification of the facts of $\varphi$ ); '-': (no relationship exists)

\begin{tabular}{|c|c|}
\hline CE1: F1 & CE6: F6 $\rightarrow(\mathrm{F} 3 \mid \mathrm{T} 1) \rightarrow(\mathrm{F} 5, \mathrm{~F} 10) \rightarrow \mathrm{T} 2$ \\
\hline CE2: F2 & CE7: F4, F11 $\rightarrow$ F5 \\
\hline CE3: $(\mathrm{F} 3 \mid \mathrm{T} 1) \rightarrow(\mathrm{F} 4, \mathrm{~F} 5, \mathrm{~F} 9) \rightarrow \mathrm{T} 2$ & CE8: F6 $\rightarrow$ F11 $\rightarrow$ F5 \\
\hline CE4: $(\mathrm{F} 3 \mid \mathrm{T} 1) \rightarrow(\mathrm{F} 4, \mathrm{~F} 5, \mathrm{~F} 10) \rightarrow \mathrm{T} 2$ & CE9: $(\mathrm{F} 3 \mid \mathrm{T} 1) \rightarrow(\mathrm{F} 7, \mathrm{~F} 8, \mathrm{~F} 9) \rightarrow \mathrm{T} 2$ \\
\hline CE5: $\mathrm{F} 6 \rightarrow(\mathrm{F} 3 \mid \mathrm{T} 1) \rightarrow(\mathrm{F} 5, \mathrm{~F} 9) \rightarrow \mathrm{T} 2$ & CE10: F7, F11 $\rightarrow$ F8 \\
\hline
\end{tabular}

Fig. 5. Contextualised executions

The next step for modelling of a contextualised business process is specification of its contextualised executions (Fig. 5). A contextualised execution is a set of fact verifications and task executions that specifies a correct execution of a business process or of a fragment of a business process for a context variant.

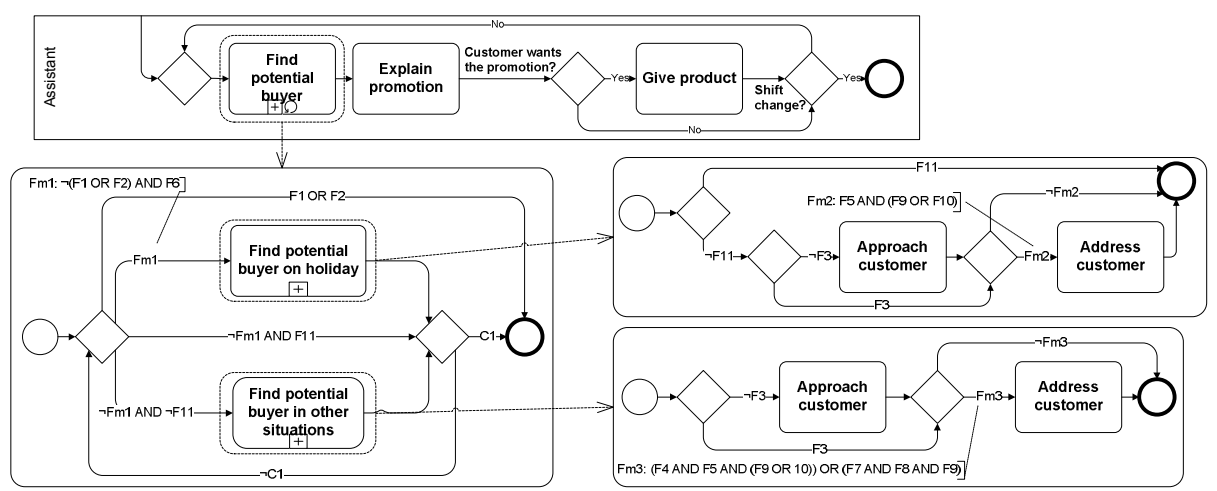

Fig. 6. Contextualised business process model 
Contextualised executions are specified by extending the final context variants of a business process with the execution sequence of its tasks (' $\rightarrow$ '). The manageable facts and their associated tasks are put in brackets and the symbol ' $\mid$ ' is put between them: either the fact is true or the task has to be executed.

Finally, a contextualised business process model is created on the basis of the constraints (fact verification and task execution sequences) that the contextualised executions impose. BPMN has been extended by labelling its sequence flows for specification of formulas that have to hold so that a sequence flow is executed. Fact and formula verification is represented by means of gateways. Fig. 6 shows the effect of contextualisation of the task "Find potential buyer" for the running example.

\section{Conclusions and Future Work}

This paper has addressed several challenges of context-aware business process modelling in order to allow research on it to further advance. As a result, an approach for business process contextualisation has been presented.

The approach adapts context analysis for analysis of business process context, and provides mechanisms and guidance for analysis of business process context and its variants and for modelling of contextualised business processes. It facilitates discovery and adequate specification of relevant context properties in the form of facts, as well as of the relationships between facts and between facts and tasks of a contextualised business process. These relationships affect business process execution. Furthermore, the mechanisms and guidance can guarantee that a contextualised business process fits its context and is sound.

As future work, we have to address approach automation and formal evaluation.

Acknowledgements. This work has been developed with the support of the Spanish Government under the projects SESAMO TIN2007-62894 and HI2008-0190 and the program FPU AP2006-02324, partially funded by the EU Commission through the projects COMPAS, NESSOS and ANIKETOS, and co-financed by FEDER. The authors would also like to thank Amit K. Chopra for his useful comments.

\section{References}

1. Ali, R., Dalpiaz, F., Giorgini, P.: A Goal-based Framework for Contextual Requirements Modeling and Analysis. Requirements Engineering Journal (to appear) (2010)

2. Hallerbach, A., Bauer, T., Reichert, M.: Capturing Variability in Business Process Models: The Provop Approach. Journal of Software Maintenance and Evolution (to appear) (2010)

3. Rosemann, M., Recker, J., Flender, C.: Contextualisation of business processes. International Journal of Business Process Integration and Management 3(1):47-60 (2008)

4. Smanchat, S., Ling, S., Indrawan, M.: A Survey on Context-Aware Workflow Adaptations. In: MoMM2008, pp. 414-417 (2008)

5. Weske, M.: Business Process Management. Springer (2007) 